\title{
Publisher's Note: Experimental Neutron Capture Rate Constraint Far from Stability [Phys. Rev. Lett. 116, 242502 (2016)]
}

S. N. Liddick, A. Spyrou, B. P. Crider, F. Naqvi, A. C. Larsen, M. Guttormsen, M. Mumpower, R. Surman, G. Perdikakis, D. L. Bleuel, A. Couture, L. Crespo Campo, A. C. Dombos, R. Lewis, S. Mosby, S. Nikas, C. J. Prokop, T. Renstrom, B. Rubio, S. Siem, and S. J. Quinn

(Q) (Received 14 March 2019; published 29 March 2019)

DOI: 10.1103/PhysRevLett.122.129902

This paper was published online on 16 June 2016 with an omission in the Acknowledgments section. On page 5, the last sentence of the Acknowledgments should read as "A. C. L. acknowledges funding through ERC-STG-2014 under Grant Agreement No. 637686." The Acknowledgments have been corrected as of 21 March 2019. The Acknowledgments are incorrect in the printed version of the journal. 